\title{
Exosomes derived from platelet-rich plasma promote the re-epithelization of chronic cutaneous wounds via activation of YAP in a diabetic rat model
}

\author{
Shang-Chun Guo ${ }^{1 *}$, Shi-Cong Tao ${ }^{2 *}$, Wen-Jing Yin ${ }^{2}$, Xin Qi ${ }^{2}$, Ting Yuan ${ }^{2}$, Chang-Qing Zhang ${ }^{1,2 \bowtie}$ \\ 1. Institute of Microsurgery on Extremities, Shanghai Jiao Tong University Affiliated Sixth People's Hospital, 600 Yishan Road, Shanghai 200233, China; \\ 2. Department of Orthopedic Surgery, Shanghai Jiao Tong University Affiliated Sixth People's Hospital, 600 Yishan Road, Shanghai 200233 , China. \\ * Co-first authors: These authors contributed equally to this work.
}

$\triangle$ Corresponding author: Prof. Chang-Qing Zhang Or Dr. Ting Yuan, Department of Orthopedic Surgery, Shanghai Jiao Tong University Affiliated Sixth People's Hospital, 600 Yishan Road, Shanghai 200233, China. E-mail: zhangcq@sjtu.edu.cn Tel: +86-21-64369181.

(1) Ivyspring International Publisher. Reproduction is permitted for personal, noncommercial use, provided that the article is in whole, unmodified, and properly cited. See http://ivyspring.com/terms for terms and conditions.

Received: 2016.07.11; Accepted: 2016.09.13; Published: 2017.01.01

\begin{abstract}
Chronic wounds have become an economic, social, and public health burden and need advanced treatment. Platelet-rich plasma (PRP) has been used extensively in treatment of chronic wounds because it contains an abundance of growth factors secreted by platelets. The exosomes derived from PRP (PRP-Exos) have been proven to encapsulate principal growth factors from platelets. This study is the first to show that these exosomes may exert the function of PRP. PRP-Exos can effectively induce proliferation and migration of endothelial cells and fibroblasts to improve angiogenesis and re-epithelialization in chronic wounds. We regulated YAP to verify the PRP-Exos-dependent effect on fibroblast proliferation and migration through YAP activation. In vivo, we observed the cutaneous healing process in chronic wounds treated with PRP-Exos in a diabetic rat model. We provide evidence of the probable molecular mechanisms underlying the PRP effect on healing of chronic ulcers and describe a promising resource of growth factors from exosomes without species restriction.
\end{abstract}

Key words: chronic wounds; platelet-rich plasma (PRP); exosomes; Yes-associated protein; re-epithelization.

\section{Introduction}

Chronic wounds cause an economic, social, and public health burden, which is rapidly increasing with the aging population in developing countries [1]. Normal healing of a skin wound requires integration of the complicated biological and molecular events of cell migration and proliferation, extra-cellular matrix (ECM) synthesis and deposition, vascularization, and remodeling $[2,3]$. In chronic wounds, all temporallyand spatially-overlapping wound healing phases are described as inhibited [4], especially in the proliferation and matrix remodeling phases.

During the proliferation phase, both cell proliferation and migration are required for the formation of granulation tissue to support epithelialization [4] but these processes are disrupted in chronic wounds [5]. During the matrix remodeling phase, re-establishment of a normal blood supply provides a favorable microenvironment for epidermal and dermal cell migration and proliferation. In turn, this leads to wound re-epithelialization and restoration of epidermal integrity. The epithelialization process is impaired in all types of chronic wounds [6]. Fibroblasts proliferate within the wound and synthesize ECM. It is known that deposition of a number of matrix components differs between chronic and acute wounds, resulting in impaired cellular proliferation and migration [7].

Conventional treatments include dressing changing and debridement, but, in many cases, these treatments do not result in reliably satisfactory outcomes [8-10]. Consequently, there has been heightened interest in developing new advanced therapies to address the compromised wound.

Specifically, in the treatment of chronic wounds, 
platelet-rich plasma (PRP) has shown promising experimental and clinical results [11]. Platelets are cytoplasmic fragments formed in the marrow and are approximately $2 \mu \mathrm{m}$ in diameter [12]. The regenerative potential of PRP is generally considered to be attributable to the supra-physiological concentrations of growth factors released from activated platelets, including platelet-derived growth factor (PDGF), transforming growth factor- $\beta$ (TGF- $\beta$ ), and vascular endothelial growth factor (VEGF) [13]. These growth factors have been demonstrated in a large number of studies to play essential roles in tissue regeneration and wound healing including neo-vascularization [14-16]. In spite of these benefits, one flaw restricting the application of PRP in the clinical setting is the requirement for autologous platelets.

Besides these growth factors, stimulation of platelets also causes them to secrete a number of extracellular vesicles, including exosomes (40 to 100 $\mathrm{nm}$ in diameter) [17]. In recent years, much interest has been devoted to exosomes [18], which act as carriers of bioactive proteins, mRNAs, and microRNAs [19] and are increasingly regarded as playing crucial roles in cell-cell [20] and platelet-cell communications [21].

Although the functions and the underlying mechanisms of exosomes derived from nucleated cells (such as cancer cells [22], immune cells [23], and stem cells [24]) have been extensively studied in recent years, few studies have assessed the existence and roles of platelet-derived exosomes. In 2014, Torreggiani et al. [17] isolated exosomes from PRP, and demonstrated their potential beneficial effect on the proliferation, migration, and osteogenic differentiation of BMSCs (bone mesenchymal stem cells). This was the first report describing the role of exosomes derived from platelets in tissue regeneration. The results suggest that PRP-derived exosomes (PRP-Exos) are important new effectors of PRP activity, and may provide an advantageous nano-delivery system to improve bone repair. However, the in vivo effect and underlying molecular mechanisms of PRP-Exos in tissue regeneration were not studied in this report, and remain unclear.

In addition to this, exosomes have the capacity to carry a large cargo load, to protect the contents from degradative enzymes or chemicals and, because of low immunogenicity and stability, they represent optimal carriers for nano-delivery treatments [25]. Above all, exosomes are not immunogenic and exhibit no species differences [26]. Thus, signals can be transmitted across species and exosomes can be obtained from other species and prepared for clinical use.
Since the growth factors secreted by platelets can be encapsulated into exosomes, VEGF and bFGF (basic fibroblast growth factor) carried by exosomes to the affected cells can promote angiogenesis in normal endothelial cells (ECs) via the PI3K/Akt signaling pathway [27]. PDGFBB then induces fibroblast proliferation through the Erk (extracellular signal regulated kinase) pathway.

Recently, our research group has focused on the Hippo/YAP (Yes-associated protein) signal pathway [28-30]. It has been reported that YAP is closely involved in skin repair and re-epithelialization [31]. It is well known that TGF- $\beta$ signaling is an important pathway controlling fibrotic events [32]. Rho GTPase (RhoA) activates downstream target proteins such as ROCK (Rho-Associated Coil Kinase) to prompt re-arrangement of the cytoskeletal elements associated with cell spreading, cell growth regulation, and cytokinesis [33], while Rho modulates ROCK to regulate YAP phosphorylation [34]. Dephosphorylation activates YAP, causing it to be transported to the nucleus where it in turn activates transcription of downstream genes to promote proliferation [35]. Moreover, according to research by Schlegelmilch et al. [31], YAP is a determinant of the proliferative capacity of epidermal stem cells and promotes their re-epithelialization.

Based on these considerations, we studied the use of PRP-Exos in chronic wound healing both in vitro and in vivo, and for the first time, outline a preliminary potential mechanism.

\section{Materials and Methods}

This study was performed in adherence with the principles of the Declaration of Helsinki. Written informed consent was obtained from each donor with the permission of the Institutional Review Board of Shanghai Sixth People's Hospital. All procedures involving the donors were approved by the ethical committee of the Shanghai Sixth People's Hospital, Shanghai Jiao Tong University School of Medicine.

The use of animals in these experiments observed the Interdisciplinary Principles and Guidelines for the Use of Animals in Research, Testing, and Education. All experimental and animal care procedures were approved by the Animal Research Ethics Committee of Shanghai Sixth People's Hospital and performed in accordance with the National Institutes of Health Guidelines for the Care and Use of Laboratory Animals.

\section{Isolation of human platelet-rich plasma (PRP)}

A comprehensive description of the derivation and characterization of PRP has been provided in our previous publications [36, 37]. Briefly, whole blood 
was collected from healthy volunteers into acid citrate dextrose solution A (ACD-A) anticoagulant at a ratio of $1 \mathrm{~mL}$ ACD-A:9 mL blood. To separate platelets from erythrocytes and leukocytes in plasma, $40 \mathrm{~mL}$ of this mixture was put into a $50-\mathrm{mL}$ centrifuge tube and centrifuged at $160 \times g$ for $10 \mathrm{~min}$, then the separated plasma containing platelets was transferred to a new centrifuge tube and centrifuged at $250 \times \mathrm{g}$ for another 15 min. Most of the supernatant plasma was discarded, before the platelet pellet was resuspended in the residual plasma to obtain $4 \mathrm{~mL}$ PRP.

\section{Exosome isolation from PRP}

Exosome isolation was based on the method of Thery et al. [38]. PRP samples were centrifuged at 250 $\times g$ for $15 \mathrm{~min}$ and the platelet pellet was washed three times with PBS (calcium-free, magnesium-free and phenol red-free; Gibco, ThermoFisher Scientific Inc., Waltham, MA, USA). The resuspended platelet pellet was activated then put through a series of low-speed centrifugation steps $(300 \times \mathrm{g}$ for $10 \mathrm{~min}$, $2,000 \times g$ for $10 \mathrm{~min}$ ) to discard cell debris. The supernatant was filtered through a $0.22 \mu \mathrm{m}$ filter (Merck-Millipore, Darmstadt, Germany) and transferred to a $15 \mathrm{~mL}$ Amicon Ultra-15 Centrifugal Filter Unit (Merck-Millipore) and centrifuged at 4,000 $\times g$. The ultrafiltered liquid was washed with PBS three times and the ultrafiltration step was repeated. To purify the exosomes, the ultrafiltered liquid was transferred onto a $30 \%$ sucrose- $\mathrm{D}_{2} \mathrm{O}$ cushion in an Ultra-Clear $^{\mathrm{TM}}$ tube (Beckman Coulter, Brea, CA, USA) and ultra-centrifuged at $100,000 \times \mathrm{g}$ for $70 \mathrm{~min}$ to pellet the exosomes, which were then washed in a large volume of PBS and ultra-centrifuged again at the same high speed for $70 \mathrm{~min}$. All centrifugations were performed at $4^{\circ} \mathrm{C}$. The exosome pellet was resuspended in sterile PBS and stored at $-80^{\circ} \mathrm{C}$ until use.

In order to compare the effect of PRP and PRP-Exos, the method was designed to prepare comparable PRP and PRP-Exos groups containing the same total protein content. Briefly, the washed PRP was centrifuged to obtain its supernatant after activating, which contained the main active constituents released from activated PRP. In this study, the supernatant of activated PRP was named PRP-AS. The protein content of PRP-AS and PRP-Exos was determined by the Pierce ${ }^{\mathrm{TM}}$ BCA Protein Assay Kit (Thermo Fisher Scientific Inc.) and standardized [39]. To observe the effect on cells in vitro, protein concentrations of $5 \mu \mathrm{g} / \mathrm{mL}$ and 50 $\mu \mathrm{g} / \mathrm{mL}$ were adopted.

\section{Identification of PRP-Exos}

To analyze the size distribution of PRP-Exos,
Nanosizer $^{\mathrm{TM}}$ technology (Malvern Instruments, Malvern, UK) was adopted. A Hitachi H-7650 transmission electron microscope (TEM) was employed to observe exosome morphologies, and the proteins encapsulated into exosomes were analyzed by classical western blotting to test for specific exosome markers, such as CD9, CD63, CD81, and the source marker CD41.

\section{Cells and cell culture}

The effect of PRP-AS and PRP-Exos on cells was studied in vitro. The human microvascular endothelial cell line HMEC-1 was purchased from the Cell Bank of the Chinese Academy of Sciences (Shanghai, China). HMEC-1 cells were cultured in MCDB131 medium (Gibco) supplemented with $10 \%$ fetal bovine serum (FBS, Gibco), $2 \mathrm{mM}$ L-glutamine (Gibco), $10 \mathrm{ng} / \mathrm{mL}$ epidermal growth factor (EGF, Sigma-Aldrich, St. Louis, MO, USA) and $1 \mu \mathrm{g} / \mathrm{mL}$ hydrocortisone (Sigma-Aldrich). Culture of primary dermal fibroblasts followed a method described previously [40]. Briefly, skin samples were sliced into small pieces and incubated in $1 \mathrm{mg} / \mathrm{mL}$ type I collagenase solution in a $10 \mathrm{~cm}$ cell culture dish overnight. The digested dermal mixture was filtered through a $70 \mu \mathrm{m}$ cell strainer (BD BioScience, San Jose, CA, USA) and cultured in high-glucose Dulbecco's modified eagle medium (DMEM, Gibco) with 10\% FBS. Cells at passage 3 to 6 were used for subsequent experiments. Both HMEC-1 cells and fibroblasts were maintained at $37^{\circ} \mathrm{C}$ in a saturated humidified atmosphere with $5 \% \mathrm{CO}_{2}$.

\section{Lentivirus infection and RNA interference}

S127A and shYAP lentivirus were purchased from Genepharma (Shanghai, China). Cell transfection was executed according to the protocol provided by the supplier. Briefly, cells were incubated in retroviral supernatant with $5 \mu \mathrm{g} / \mathrm{mL}$ polybrene for 24 hours. Forty-eight hours after infection, cells were selected with $2.5 \mu \mathrm{g} / \mathrm{mL}$ puromycin (Sigma-Aldrich) in culture medium. The shRNA sequences used in the present study were as follows:

shYAP\#1:

5'-CTGGTCAGAGATACTTCTTAA-3'; ShYAP\#2:

5'-AAGCTTTGAGTTCTGACATCC-3';

\section{Inhibitor and agonist}

Y-27632 $2 \mathrm{HCl}$ was purchased from Selleck (Houston, TX, USA). Fibroblasts were cultured in conditioned medium containing the specified concentration of PRP-Exos $(50 \mu \mathrm{g} / \mathrm{mL})$ or Y-27632 $2 \mathrm{HCl}(20 \mu \mathrm{M})$. After treatment, cell proliferation, gene expression analysis, and western blotting were performed. 


\section{Cell proliferation}

A cell viability assay (Cell Counting Kit- 8 (CCK-8); Dojindo Molecular Technologies, Inc., Kumamoto, Japan) was used to observe the effect of PRP-Exos on the proliferation of HMEC-1 cells, as well as on fibroblasts treated with Y-27632 $2 \mathrm{HCl}$, or transfected with shYAP or S127A as described previously [30]. Briefly, the cells were plated into 96-well plates at an initial density of 5,000 cells/well and cultured at $37^{\circ} \mathrm{C}$ in $5 \mu \mathrm{g} / \mathrm{mL}$ or $50 \mu \mathrm{g} / \mathrm{mL}$ PRP-Exos[39]. Cells treated with an equal protein content of PRP-AS served as controls, and plates without cells served as the blank. On day $0,1,3,5$ and 7, $20 \mu \mathrm{L} \mathrm{CCK}-8$ solution and $180 \mu \mathrm{L}$ fresh culture medium was added to each well at each time point and incubated for $1 \mathrm{~h}$ at $37^{\circ} \mathrm{C}$. The absorbance of the wells was measured at $450 \mathrm{~nm}$ using a spectrophotometric microplate reader (Bio-Rad 680, Bio-Rad, Hercules, CA, USA). The optical density (OD) of the test wells minus the absorbance of the blank wells represented the survival/proliferation of cells.

\section{Preparation of PRP-Exos-loaded sodium alginate hydrogel}

Sodium alginate (SA) was purchased from Sigma-Aldrich, and hydrogel preparation was performed according to the procedure of Dar et al. [41]. Briefly, to prepare PRP-Exos-loaded hydrogel, a weighed quantity of SA dry powder $(1.25 \% \mathrm{w} / \mathrm{v})$ was mixed in distilled water and agitated for $1 \mathrm{~h}$ to dissolve, after which PRP-Exos (1\% v/v) were added to the solution under gentle agitation. The resultant mixtures were then poured into a 24-well plate filled with calcium chloride solution $(5 \% \mathrm{w} / \mathrm{v})$, then kept at RT for $48 \mathrm{~h}$ to gel. Next, the redundant calcium chloride solution was discarded from the well and the PRP-Exos-loaded sodium alginate hydrogel (SAH) samples were air-dried at RT. The resultant PRP-Exos-loaded SAH formed discs of $1.8 \mathrm{~cm}$ diameter and nearly $2.0 \mathrm{~mm}$ thickness.

\section{In vitro study of PRP-Exos release}

To study the release profile of PRP-Exos, an experiment was designed to evaluate the number of exosomes at a range of time-points up to $96 \mathrm{~h}$. First, PRP-Exos-loaded SAH samples were placed into a 24-well plate, and immersed in serum-free MesenGro hMSC medium ( $1 \mathrm{~mL} /$ well; MGro-500; StemRD; CA, USA) at $37^{\circ} \mathrm{C}$. The samples were then withdrawn at fixed time-points $(1,3,6,12,24,48,72$ and 96 h). All the samples extracted at each time-point, including the final $96 \mathrm{~h}$ samples, were stored at $-80^{\circ} \mathrm{C}$ until all samples were collected. The samples were then quantified using an ExoELISA CD63 Kit (System
Biosciences, Uden, The Netherlands) according to the manufacturer's instructions, and absorbance was read by a microtiter plate reader at $450 \mathrm{~nm}$ to observe the OD of the exosomes at each experimental interval. All samples were analyzed in triplicate wells in each experiment, and each experiment was repeated three times. Comparing the OD value to the standard curve, the exosome number could be calculated according to the standard curve correlation function. Therefore, the PRP-Exos release profile could be described in terms of exosome number in the medium at any given time.

\section{HMEC-1 migration and vessel-like formation assays}

The effect of the PRP-Exos on HMEC-1 migration was carefully analyzed using a transwell assay. Briefly, $1 \times 10^{4}$ cells were plated into the upper chamber of a 24-well transwell plate (Corning, Corning, NY, USA; pore size $=8 \mu \mathrm{m}$ ). Then 700 $\mu \mathrm{L} /$ well containing 5 or $50 \mu \mathrm{g} / \mathrm{mL}$ of PRP-AS or PRP-Exos were added to the lower chamber. After incubation for six hours, the cells from the upper surface of the filter membranes were removed with a cotton swab. Cells that migrated to the lower surface of the filter membrane were stained with $0.5 \%$ crystal violet for $1 \mathrm{~min}$. Migratory activity was evaluated by observing the stained cells under an optical microscope.

To investigate the capillary-like construction activity of HMEC-1 cells, the formation of vessel-like structures in ECM gel (Sigma-Aldrich) was assessed. Briefly, $50 \mu \mathrm{L}$ of cold ECM gel per well was transferred with a pre-cooled tip to a 96-well plate. After gelling for $15 \mathrm{~min}$, the ECM gel was overlaid with $100 \mu \mathrm{L}$ of a suspension of HMEC- 1 cells $\left(1 \times 10^{4}\right.$ cells/well) treated with or without the PRP-Exos. Tube formation capacity was assessed by observing the polygonal structures formed $6 \mathrm{~h}$ after seeding the cells onto the ECM gel using a light microscope.

\section{YAP nuclear localization}

Fibroblasts were seeded into a confocal glass-bottomed 6-well-plate (SPL, Gyeonggi-do, Korea). Confluent cells were left untreated, incubated with PRP-Exos $(50 \mu \mathrm{g} / \mathrm{mL})$ alone, or stimulated with Y-27632 $2 \mathrm{HCl}(20 \mu \mathrm{M})$ and PRP-Exos $(50 \mu \mathrm{g} / \mathrm{mL})$ for three hours. YAP was detected by immunofluorescence (IF) analysis.

\section{Preparation of nuclear and cytosolic extracts}

As described in our previous report [30], nuclear and cytoplasmic extracts were prepared step by step from the cultured cells using an NE-PER nuclear and cytoplasmic extraction reagents kit (Pierce Biotechnology, Rockford, IL, USA). The protein concentrations were determined using a BCA Protein 
Assay Kit (Pierce). Aliquots of the extracts were then analyzed by western blotting.

\section{Quantitative real-time PCR (qRT-PCR)}

Total RNA was extracted with TRIzol Reagent (Invitrogen, Carlsbad, CA, USA) and cDNA was synthesized from $1 \mu \mathrm{g}$ of total RNA using a TransScript All-in-One First-Strand cDNA Synthesis SuperMix (Transgen Biotech, Beijing, China). Next, qRT-PCR was performed progressively using FastStart Universal SYBR Green Master Mix (Roche, Indianapolis, IN, USA) in an ABI PRISM ${ }^{\circledR} 7900 \mathrm{HT}$ System (Applied Biosystems, Foster City, CA, USA). Beta-actin was used for normalization of the results. Primers used in the present study are as described previously [30].

\section{Western blot analysis.}

Lysates of cells or exosomes were diluted at a ratio of $1: 5$ with protein loading buffer $(6 \times)$ and heated at $95^{\circ} \mathrm{C}$ for 5 minutes. Protein extracts were separated by $10 \%$ sodium dodecyl sulfate-polyacrylamide gel electrophoresis (SDS-PAGE) at $120 \mathrm{~V}$ for 1 hour and blotted onto a polyvinylidene di-fluoride (PVDF) membrane (Merck-Millipore) for $90 \mathrm{~min}$ at $200 \mathrm{~mA}$. The membranes were then blocked for 120 min with $5 \%$ non-fat dried milk in TBST (Tris-buffered saline, 10 $\mathrm{mM}$ Tris- $\mathrm{HCl} \mathrm{pH} 7.5,150 \mathrm{mM} \mathrm{NaCl}, 0.1 \%$ Tween-20). Subsequently, the membranes were incubated with primary antibodies at $4^{\circ} \mathrm{C}$ overnight, followed by incubation with horseradish peroxidase (HRP)-labeled secondary antibodies (Cell Signaling Technology, Danvers, MA, USA) at $37^{\circ} \mathrm{C}$ for 1 hour. The immunoreactive bands were visualized using enhanced chemiluminescence reagent (Thermo Fisher Scientific) and imaged using an Image Quant LAS 4000 mini bio-molecular imager (GE Healthcare, Uppsala, Sweden).

\section{Antibodies for western blotting}

The primary antibodies used were anti-VEGF and anti-PDGFBB (Abcam, Cambridge, UK); anti-CD9, anti-CD63, anti-CD81 (System Biosciences); anti-CD41, anti-TGF- $\beta$, anti-bFGF, anti-YAP and phosphorylated YAP (p-YAP), anti-Erk1/2 and phosphorylated Erk1/2 (p- Erk1/2), anti-Akt and phosphorylated Akt (p-Akt) (Cell Signaling Technology).

\section{Preparation of diabetic rat model and experimental groups}

The ability of PRP-Exos to promote wound healing in full-thickness skin defects was investigated in a diabetic rat model. After PRP was prepared for animal experiments, the quantity of PRP-AS was determined using the Pierce ${ }^{\mathrm{TM}}$ BCA Protein Assay Kit (Thermo Fisher Scientific Inc.) standardized by protein content. According to the wound sizes, the PRP volume to be encapsulated in SAH was evaluated accurately based on the determined PRP-AS quantity. The quantity of PRP-Exos to be applied in vivo was calculated on the basis of the determined PRP-AS protein concentration and mixed with SAH, so as to obtain an equal content of protein. For comparison, SAH, PRP and untreated wounds were used as controls [11].

Thirty-six male Sprague-Dawley (SD) rats (12 weeks old; 300-400 g) were used in the present study. Freshly-prepared streptozotocin (STZ; Sigma-Aldrich) was used to induce diabetes by a single intraperitoneal injection $(55 \mathrm{mg} / \mathrm{kg}$ ) [42]. STZ was prepared in a $0.1 \mathrm{M}$ phosphate-citrate buffer ( $\mathrm{pH} 4.5)$. Rats were examined on the third day following STZ administration to confirm initiation of the induction of diabetes. Blood samples were taken from the tail vein of SD rats, and blood glucose levels $>250 \mathrm{mg} / \mathrm{dL}$ (13.9 mmol/L) measured using a glucometer (Roche) were regarded as indicating diabetes in this study. The rats' blood glucose levels and weight changes were measured every day for two weeks after diabetes induction. The study was conducted once the induction of diabetes was confirmed in the rat model.

Anesthesia was administered by intraperitoneal injection of $3 \%$ pentobarbital sodium solution (Sigma-Aldrich) at a dosage of $1.0 \mathrm{~mL} / \mathrm{kg}$ before operation. Defect areas were marked precisely with a pen and standardized full-thickness skin wounds (diameter $=1.8 \mathrm{~cm}$ ) were prepared by resecting the dorsum under aseptic conditions.

Thirty-six rats were randomly divided into 4 groups as follows: (1) Control $(n=9)$, untreated skin defects; (2) SAH ( $=9$ ), defects treated with SAH; (3) PRP $(\mathrm{n}=9)$, defects treated with PRP-loaded SAH; and (4) PRP-Exos ( $\mathrm{n}=9)$, defects treated with PRP-Exos incorporated into SAH. After the wound beds were treated by one of the four different methods, the rats received a pressure dressing to protect the wound. At 3, 7 and $14 \mathrm{~d}$ after operation, 9 rats were sacrificed and skin specimens were harvested. Skin samples were first analyzed by measuring any reduction in wound size, then micro-computed tomography (micro-CT) was used for analysis. Finally, every skin sample was analyzed by histological, immunohistochemical (IHC) and IF methods.

\section{Measurement of wound closure}

After operation, the wounds were photographed at $0,3,7$ and $14 \mathrm{~d}$ with a digital camera (Nikon, Tokyo, Japan). The margin of each wound was traced and the 
wound area was measured using ImageJ (National Institutes of Health, Bethesda, MD, USA).

The size of wound closure was calculated using the formula (1):

Percent wound size reduction $=\left[\left(\mathrm{A}_{0}-\mathrm{A}_{\mathrm{t}}\right) / \mathrm{A}_{0}\right] \times$ 100 , where $A_{0}$ is the initial wound area $(t=0)$ and $A_{t}$ is the wound area at each time interval.

\section{Microfil perfusion and micro-CT}

To evaluate blood vessel regeneration, the rats were perfused with Microfil (Microfil MV-122; Flow Tech, Carver, MA, USA) after they were euthanized at $14 \mathrm{~d}$ post-operation. Briefly, the hair of the chest was shaved and the rib cage was opened with a pair of scissors. Then, $100 \mathrm{~mL}$ of heparinized saline and 20 $\mathrm{mL}$ of Microfil were continuously perfused at a rate of $2 \mathrm{~mL} / \mathrm{min}$. The specimens were laid flat at $4^{\circ} \mathrm{C}$ in the refrigerator overnight to ensure complete polymerization. Then the wounds were scanned by micro-CT (Skyscan 1176 high-resolution in vivo micro-CT scanner; Bruker, Billerica, MA, USA) at a resolution of $9 \mu \mathrm{m}$ to examine new blood vessels. Three-dimensional images were reconstructed with the CTVol program (Bruker). The area and number of blood vessels in the defect were also determined with the software ImageJ.

\section{Histologic, IHC and IF analysis}

The rats were sacrificed at day 7 and 14 after operation and tissue containing the wound bed and surrounding healthy skin was collected for further investigation. The samples were fixed in formalin, dehydrated through a graded series of ethanol and embedded in paraffin. Sections ( $\sim 5 \mu$ thick) were stained with $\mathrm{HE}$ and Masson's trichrome and examined under an optical microscope. The neo-epithelium length was assessed according to a method described previously [43]. IHC and IF staining of the thin sections was performed to study angiogenesis during the wound-healing process. IHC staining for CD31, a marker of ECs (1:100, Abcam) and IF staining for CD31 (1:50, Abcam) and alpha-smooth muscle actin (a-SMA), a marker of vascular smooth muscle cells (1:200, Abcam), were used to estimate the newly-formed capillaries and arterioles.

For IHC staining, the sections were rehydrated, treated to retrieve the antigen and incubated with the primary antibody at $4^{\circ} \mathrm{C}$ overnight. Subsequently, the biotinylated secondary antibody and avidin-biotin-peroxidase complex were applied and the DAB substrate was used to visualize the stained sections. Then the sections were counter-stained with hematoxylin, mounted and analyzed under a light microscope.
For IF staining, the sections were rehydrated, blocked with $1.5 \%$ goat serum (Merck-Millipore), and incubated in the primary antibody overnight at $4^{\circ} \mathrm{C}$. After treating the specimens with Alexa Fluor 488and Cy3-conjugated secondary antibodies, DAPI (Sigma-Aldrich) was used to stain the nuclei. The images were examined with an LSM-880 confocal-microscopes (Carl Zeiss, Oberkochen, Germany).

To quantitate the number of blood vessels, six sections from different tissue specimens were prepared for each treatment group at each time-point. For each section, three high-power fields, containing the entire portion of the defects, were randomly sampled and the newly-formed blood vessels were estimated.

\section{Antibodies for IHC or IF}

IHC staining for CD31 was performed using an anti-CD31 primary antibody (1:100). IF staining was performed using an anti-CD31 primary antibody (1:50) and anti-a-SMA primary antibody (1:200), (all from Abcam).

\section{Statistical analysis}

All data are presented as means \pm standard deviation (SD). Independent-sample t-tests were used to compare means between two different groups. One-way analysis of variance (ANOVA) was used to determine the level of significance with GraphPad Prism software and $P$ values $<0.05$ were considered to be statistically significant.

\section{Results}

\section{Characterization of PRP-Exos}

Transmission electron microscopy (TEM), dynamic light scattering (DLS) analysis, and western blotting were used to characterize the purified nanoparticles derived from PRP. The sizes of PRP-Exos were directly tracked using a DLS system named the Nanosizer system with a size ranging from 40 to $100 \mathrm{~nm}$ (Fig. 1A). In TEM experiments with PRP-Exos, the results showed that the vast majority of these nanoparticles exhibited a cup- or sphere-shaped morphology (Fig. 1B), indicating the presence of exosomes. Western blotting showed the presence of exosome markers, such as CD9, CD63 and CD81 and the platelet marker CD41 (Fig. 1C), which further confirmed their identity as exosomes. All these data suggested that these nanoparticles were actually exosomes. At the same time, the exosome cargo was confirmed to contain PDGFBB, TGF- $\beta$ (TGFB1) and bFGF in PRP-Exos and the protein expression levels were higher than those in PRP-AS (Fig. 1C) when the total protein levels were equal. In addition, VEGF was 
also contained in PRP-Exos.

\section{Evaluation of cellular responses to PRP-Exos}

The proliferation of fibroblasts and HMEC-1 cells cultured for $0,1,3,5$ and $7 \mathrm{~d}$ in different concentrations of PRP-Exos is shown in Fig. 2A and 2B. The PRP-Exos had a considerable effect on cell proliferation throughout the healing process. Moreover, fibroblasts cultured in PRP-Exos proliferated significantly better than those cultured in PRP-AS at all time-points tested, while HMEC-1 cell proliferation showed no significant difference between the $50 \mu \mathrm{g} / \mathrm{mL}$ PRP-AS and the $5 \mu \mathrm{g} / \mathrm{mL}$ PRP-Exos groups at day 7. For fibroblasts, at days 3 and 5 , the $5 \mu \mathrm{g} / \mathrm{mL}$ exosomes and $50 \mu \mathrm{g} / \mathrm{mL}$ exosome groups showed similar proliferation rates.

Transwell assays showed that the PRP-Exos significantly promoted migration of fibroblasts and HMEC-1 cells compared to control or PRP-AS (Fig. 2C-D). These data indicated that cells in the PRP-Exos group migrated much further than those in the PRP-AS group (Fig. 2C-D) at the same total protein level.

Assays of tubule formation activity of the HMEC-1 cells were used to determine the angiogenesis-promoting potential of the PRP-Exos. After incubation on ECM gel for 6 hours, HMEC-1 cells incubated with PRP-Exos were found to have formed elongated, vessel-like structures, whereas HMEC-1 cells treated with PRP-AS formed incomplete or sparse tubular networks (Fig. 2C). In promoting angiogenesis, the effect of PRP-Exos was shown to be more potent than that of PRP-AS.

\section{Involvement of RhoA/YAP signaling in the PRP-Exos-induced proliferation and re-epithelialization responses of fibroblasts.}

After treating the fibroblasts for three hours with PRP-Exos or PRP-AS, western blotting was performed to detect YAP de-phosphorylation. When YAP is dephosphorylated, it is transported to the nucleus where it activates downstream factors. CTGF (connective tissue growth factor), one downstream effector protein of YAP [44], promotes fibroblast proliferation and collagen deposition [45] (Fig. 3A). To verify whether YAP was the critical mediator of PRP-Exos-dependent modulation in the proliferation and migration of fibroblasts, we first used lentiviral shRNA to knock down the level of YAP in fibroblasts and confirmed the inhibitory efficiency of the shRNA (Fig. 3B). We also found that the PRP-Exos-induced promotion of fibroblast proliferation and migration was blocked (Fig. 3C-D). Furthermore, we over-expressed S127A, which is a mutant of YAP that generates a constitutively-active YAP protein in fibroblasts. After transfection, we analyzed the proliferation of fibroblasts transfected with S127A or its empty vector at day $0,1,3,5$, and 7 using the CCK-8 kit assay and analyzed migration with transwell assays, and found that the promotional effects of PRP-Exos on fibroblast proliferation and migration were mimicked by S127A over-expression (Fig. 3E-F). All these results indicate that the PRP-Exos-induced promotion of fibroblast proliferation and migration can be attributed to YAP activation.

$\mathrm{Y}-276322 \mathrm{HCl}$ is a selective ROCK inhibitor [46]. Addition of Y-27632 $2 \mathrm{HCl}$ to fibroblasts treated with PRP-Exos increased the phosphorylation level of YAP (Fig. 3G). However, the protein expression of YAP in the nucleus (Fig. 3H), as well as YAP nuclear localization, (Fig. 3I) were both reduced. As shown in Fig. 3G-I, we found that the PRP-Exos-induced YAP activation was blocked by $\mathrm{Y}-276322 \mathrm{HCl}$. This resulted in blocking of the PRP-Exos-induced promotion of proliferation and migration in fibroblasts (Fig. $3 \mathrm{~J}-\mathrm{K}$ ). All these results indicate that the activating effect of PRP-Exos on YAP was effected through the Rho-ROCK axis [30].
A

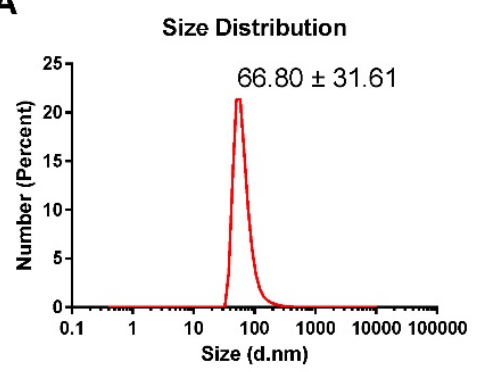

B

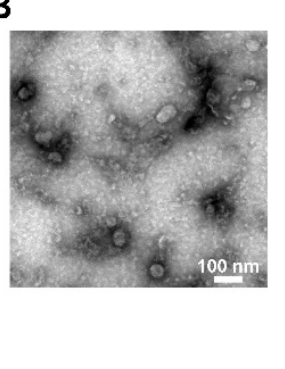

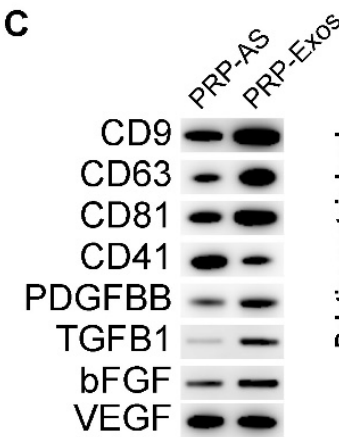

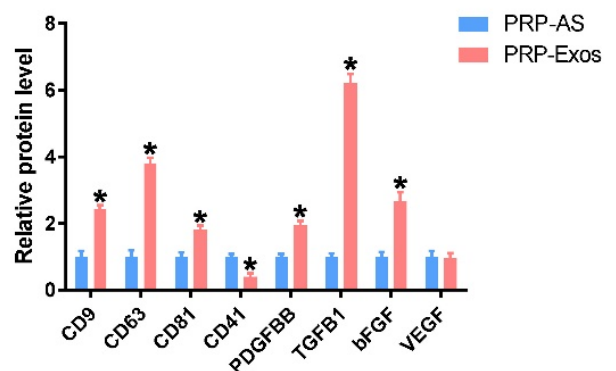

Figure 1. Characterization of PRP-Exos: (A) Particle size distribution measured by DLS. (B) Morphology observed by TEM. (C) Western blotting and quantitative analysis of the exosome surface markers and cargo. Scale bar: $100 \mathrm{~nm}$. $* P<0.05$ compared with PRP-AS 
A

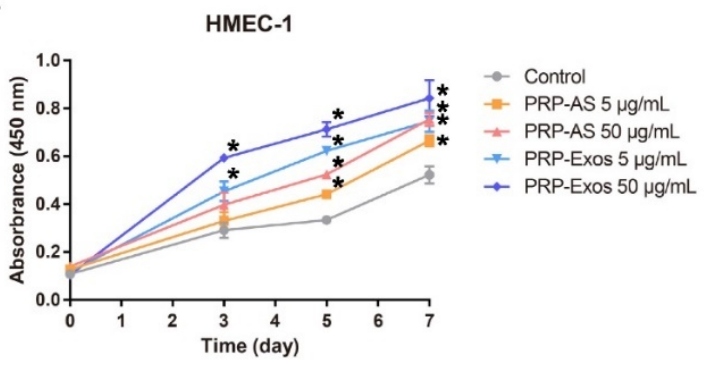

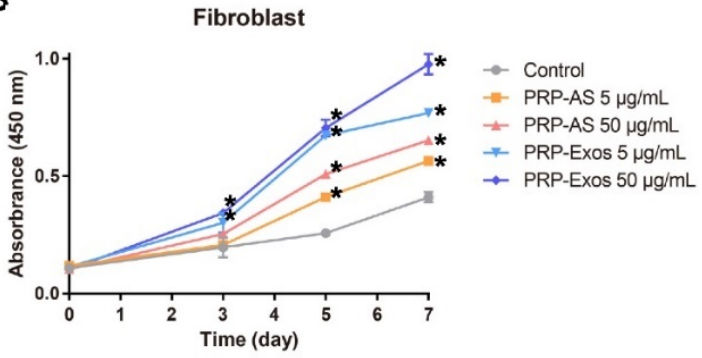

C
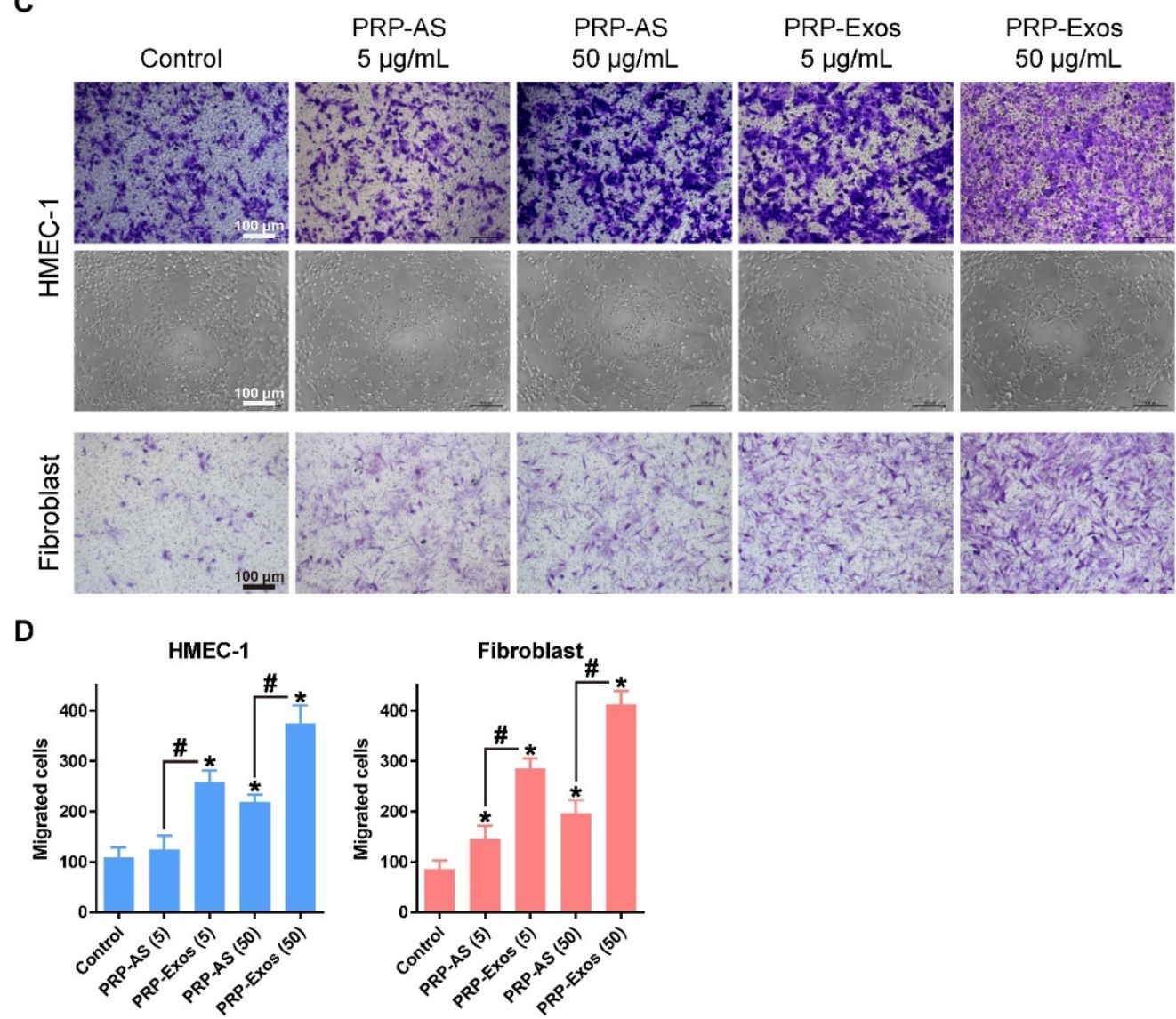

Figure 2. (A-B) PRP-Exos enhanced the proliferation of HMEC-1 cells and fibroblasts when analyzed by CCK-8 assay with different concentration of PRP-Exos and PRP-AS. (C) Representative photomicrographs showing the effect of different concentrations of PRP-Exos and PRP-AS on the transwell migration (violet-stained cells) of HMEC-1 cells and fibroblasts, and tubule formation of HMEC-1 cells after incubation for $6 \mathrm{~h}$. Scale bar: $100 \mu \mathrm{m}$. (D) Quantitative analysis of the transwell assays. *P< 0.05 compared with control. $\# P<0.05$ comparing PRP-AS and PRP-Exos.

Thus, we demonstrate that the stimulatory effects of PRP-Exos on the proliferation and migration of fibroblasts mainly resulted from activation of the Rho-YAP signal pathway.

\section{PI3K/Akt and Erk1/2 signaling are involved in exosome-induced angiogenesis-promoting and proliferation effects.}

Western blotting results showed that VEGF, bFGF and PDGFBB were encapsulated in the PRP-Exos (Fig. 1C), indicating that both the PI3K/Akt and Erk pathways may be activated by the exosomes. To verify this hypothesis and determine which effector proteins respond to PRP-Exos stimulation, western blotting was carried out to assess the protein levels of Akt and p-Akt, Erk and p-Erk in HMEC-1 following treatment with PRP-Exos or PRP-AS for 30 min. Compared to PRP-AS, incubation with PRP-Exos resulted in a significant increase in phosphorylation of Akt and Erk (Fig. 3L), indicating that PI3K/Akt and Erk signaling was also activated by the exosomes in HMEC-1. Activation of the PI3K/Akt and Erk pathway might be the underlying mechanism through which PRP-Exos exert pro-angiogenic and proliferation-inducing effects on ECs. 


\section{A}

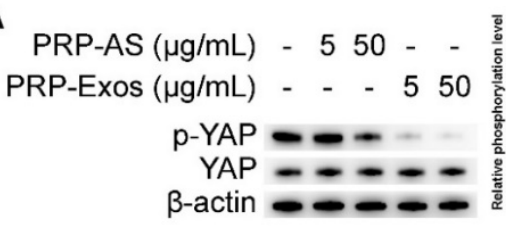

$\beta$-actin

PRP-AS $(\mu \mathrm{g} / \mathrm{mL}) \quad-550-\quad-$

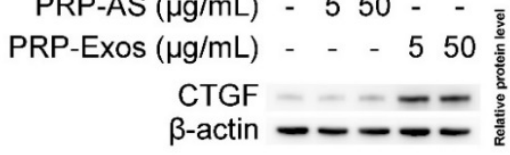

D

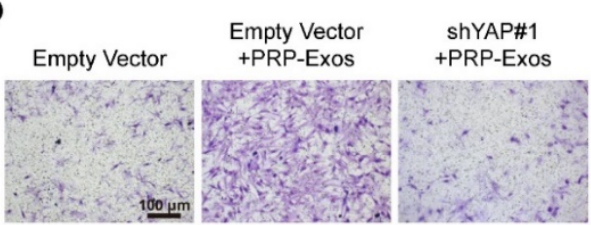

$\mathbf{F}$

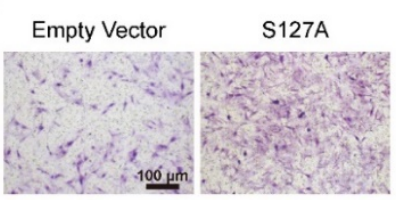

G

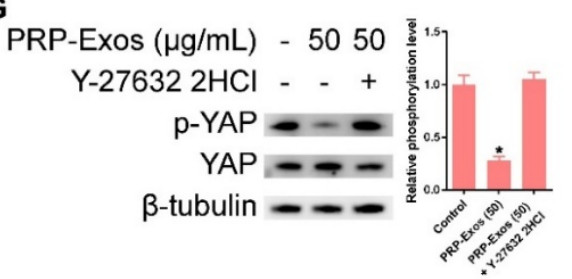

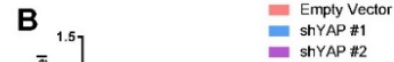

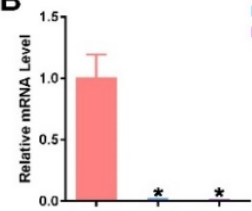

C

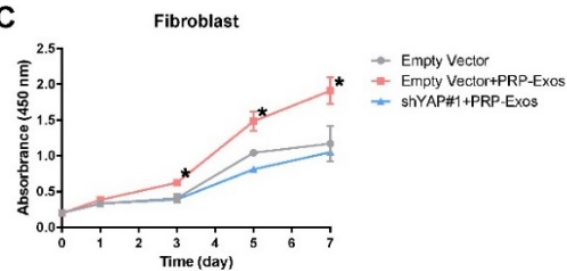

E

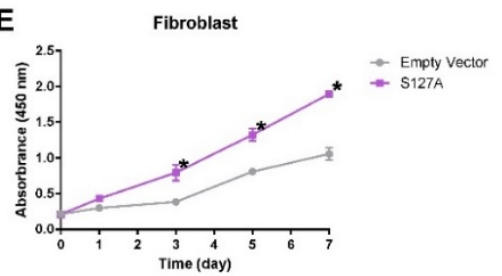

I

H

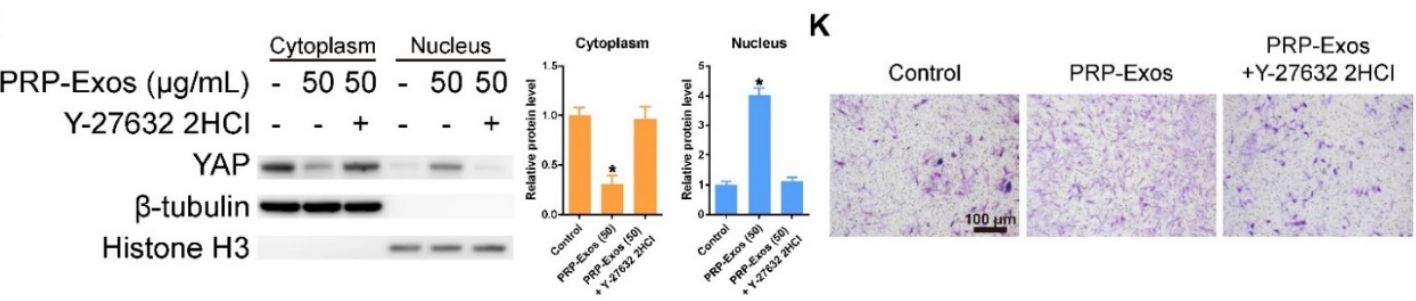

L

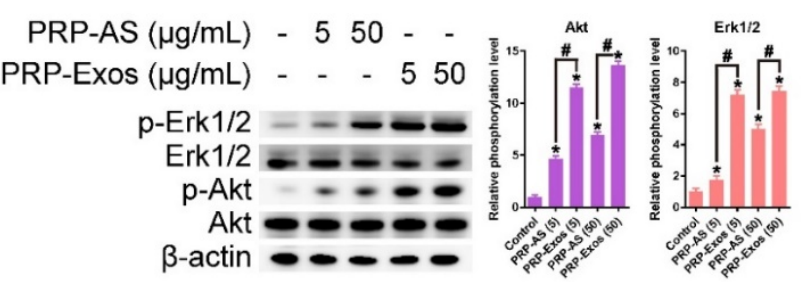

Figure 3. (A) Western blotting showed that exosome-treated fibroblasts induced significantly greater YAP de-phosphorylation and much higher CTGF expression compared with the other three groups, indicating that the Rho/YAP signaling pathway was activated in fibroblasts treated with PRP-Exos and PRP. $* P<0.05$ compared with controls. \#P $<$ 0.05 comparing PRP-AS and PRP-Exos. (B) YAP expression in fibroblasts after treatment with shYAP. $* P<0.05$ compared with empty vector. (C) Proliferation of fibroblasts transfected with shYAP or its empty vector and treated with PRP-Exos, analyzed by cck-8 assay. *P< 0.05 compared with empty vector. (D) Migration of fibroblasts transfected with shYAP or its empty vector and treated by PRP-Exos, analyzed by transwell assay, scale bar: $100 \mu \mathrm{m}$. (E) Proliferation of fibroblasts transfected with S127A or its empty vector then treated with PRP-Exos and analyzed by cck- 8 assay. $* P<0.05$ compared with empty vector. (F) Migration of fibroblasts transfected with S127A or its empty vector and treated with PRP-Exos, analyzed by transwell assay, scale bar: $100 \mu \mathrm{m}$. (G) YAP de-phosphorylation following treatment with PRP-Exos was blocked by $Y$ - $276322 \mathrm{HCl}$. $* P<0.05$ compared with control. $(\mathrm{H})$ YAP protein expression in the cytoplasm and nucleus of fibroblasts treated with PRP-Exos was altered by $\mathrm{Y}-276322 \mathrm{HCl}$. $* \mathrm{P}<0.05$ compared with control. (I) YAP nuclear localization in fibroblasts treated with PRP-Exos was blocked by Y-27632 $2 \mathrm{HCl}$. Scale bar: $50 \mu \mathrm{m}$. (J) Proliferation of fibroblasts treated with PRP-Exos was blocked by $\mathrm{Y}-276322 \mathrm{HCl}$, as shown by cck-8 assay. $* P<0.05$ compared with control. (K) Migration of fibroblasts treated with PRP-Exos was blocked by $\mathrm{Y}-276322 \mathrm{HCl}$, as shown by transwell assay. Scale bar: $100 \mu \mathrm{m}$. (L) Erk and Akt phosphorylation level in fibroblasts treated with PRP-Exos or PRP-AS. $* P<0.05$ compared with control. \#P $<0.05$ comparing PRP-AS and PRP-Exos. 
A

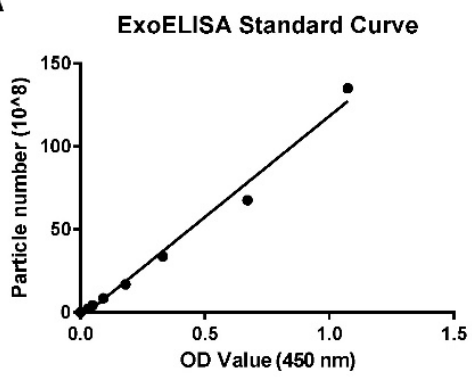

Releasing Curve

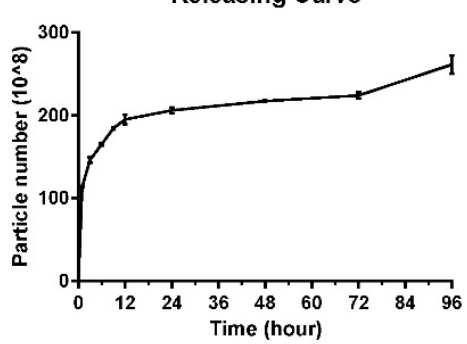

C

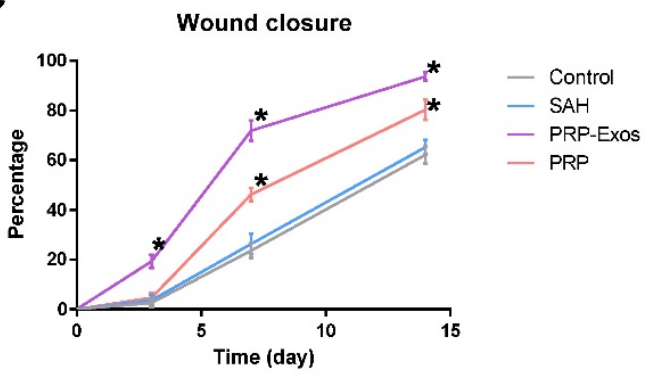

B

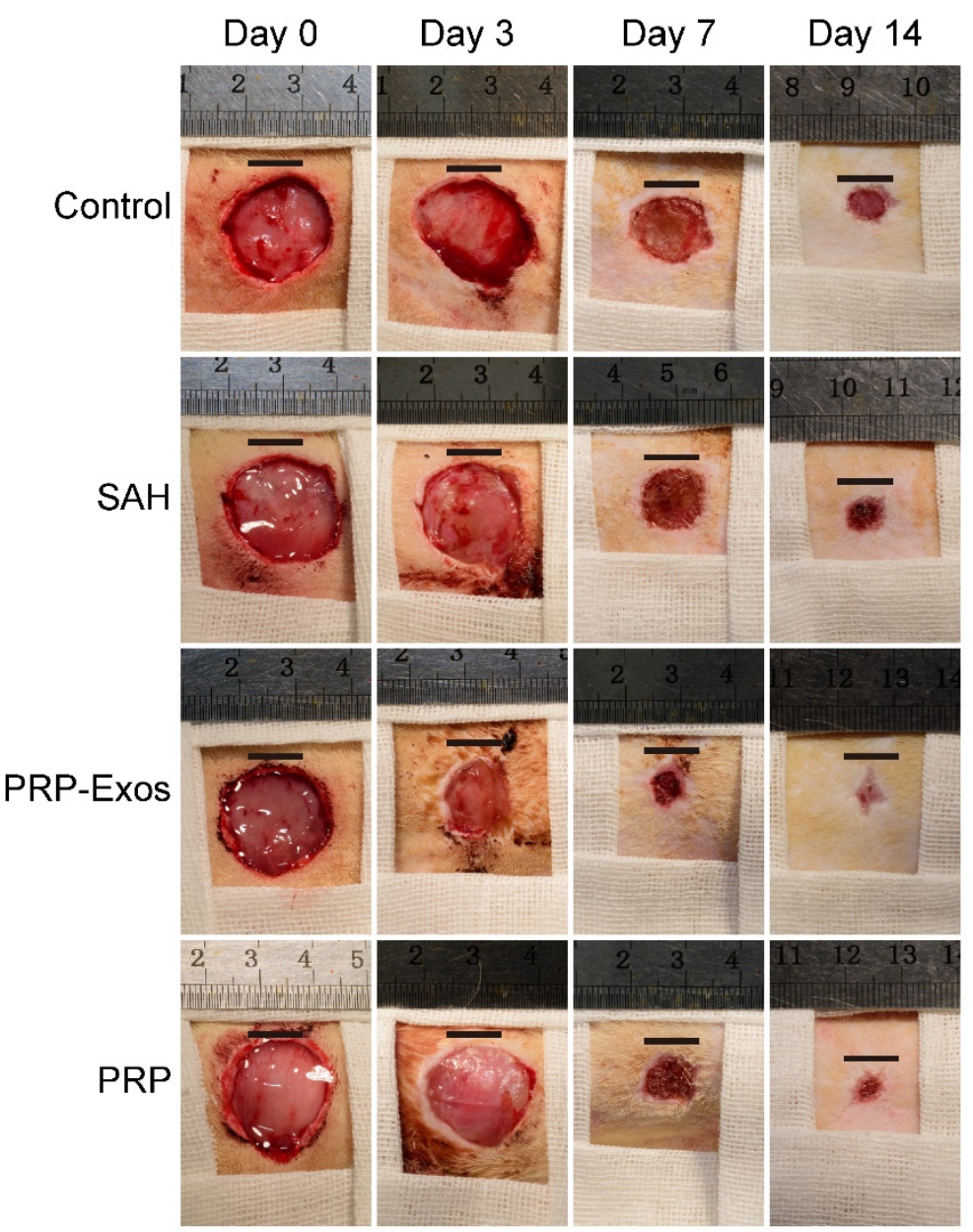

Figure 4. (A) Cumulative amount of PRP-Exos released from SAH into serum-free MesenGro hMSC medium as a function of immersion time. (B) Representative images of full-thickness skin defects in a diabetic rat model, left untreated (control) or treated with SAH, PRP or PRP-Exos, at 0, 3, 7 and 14 days after operation. Scale bar: 10 mm. (C) Percentage wound closure of untreated defects and defects treated with SAH, PRP or PRP-Exos at 3,7 and 14 days after surgery. $* P<0.05$ compared with control.

\section{Release of PRP-Exos from SAH.}

Detection of the release of PRP-Exos from SAH was carried out using an ExoELISA CD63 kit. The release of exosomes was detected at 1, 3, 6, 9, 12, 24, 48,72 and $96 \mathrm{~h}$. The results showed that the PRP-Exos can be found in the SAH leaching solution and the release of PRP-Exos increased rapidly with increasing immersion time (Fig. 4A). The release platform appeared at $12 \mathrm{~h}$.

\section{Evaluation of wound healing treated with PRP-Exos in vivo}

No adverse effects were observed at any time during the experimental procedure (Fig. 4B). Digital photographs showed the progress in closure of the untreated wounds and those treated with PRP-Exos, PRP and SAH at $0,3,7$ and 14 days. While the wounds in all four groups contracted over time, the wound size in the PRP-Exos group was smaller than that of the untreated, SAH or PRP groups. In particular, the 
wounds treated with PRP-Exos had almost closed at day 14 whereas the untreated had not closed.

Quantification of the wound closure confirmed that the wounds treated with PRP-Exos closed significantly faster than the untreated, SAH-treated or PRP-treated wounds at all four time-points (day 0, 3, 7 and 14) (Fig. 4C). The wounds treated with PRP showed significantly better healing at days 7 and 14 compared to the untreated or SAH group wounds. Even though the PRP-Exos provided better wound closure than the PRP, there was no significant difference between these two groups at day 14 .

\section{Micro-CT evaluation of wound healing}

Fourteen days after surgery, the formation of blood vessels in the wounds treated with PRP-Exos, $\mathrm{PRP}$, or SAH and the untreated wounds were examined by micro-CT. The reconstructed three-dimensional images (Fig. 5A) showed a much higher density of blood vessels in the defects treated with PRP-Exos than in those treated with either PRP or $\mathrm{SAH}$, or in the untreated defects. Quantification of the newly-formed blood vessels showed a significantly higher blood vessel area and blood vessel number in the defects treated with PRP-Exos than in the PRP-treated, SAH-treated or the untreated defects (Fig. 5B).

\section{Histologic, IHC and IF analyses}

Light micrographs of HE-stained sections show the neo-epithelium in the untreated wounds and those treated with PRP-Exos, PRP, and SAH at 14 days (Fig. 5C). The width of the photograph indicates the total initial wound size $(1.8 \mathrm{~cm})$. The entire epithelium in the wounds treated with PRP-Exos appeared significantly better than that in the untreated wounds at 7 days after operation and the healed epithelium was significantly longer than that of the untreated wounds at day 14 (Fig. 5D). While the neo-epithelium length of the wounds treated with the PRP-Exos was longer than that of the untreated wounds at day 14, the difference between the PRP-Exos and PRP treatment groups was also significant. Histological analysis of Masson's
A

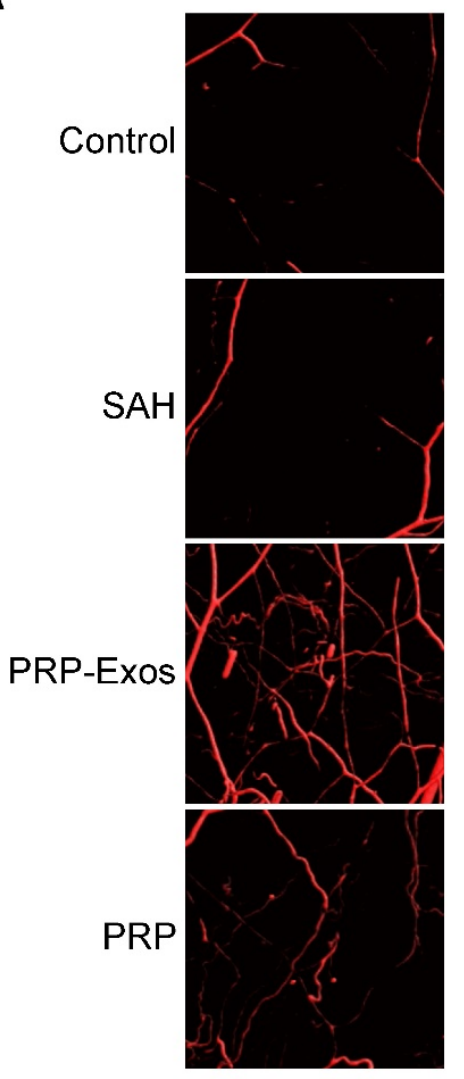

B
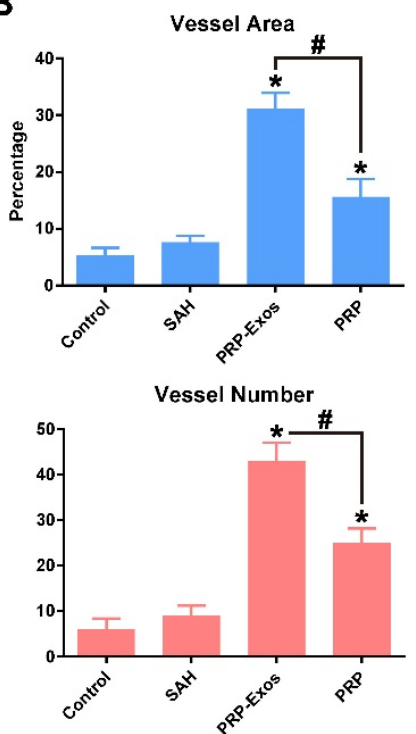

D

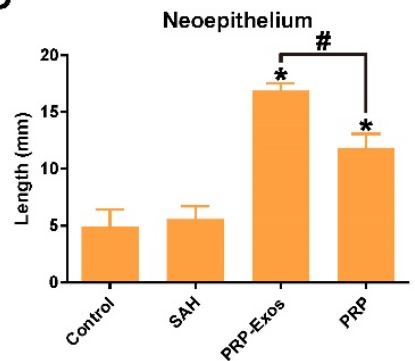

C
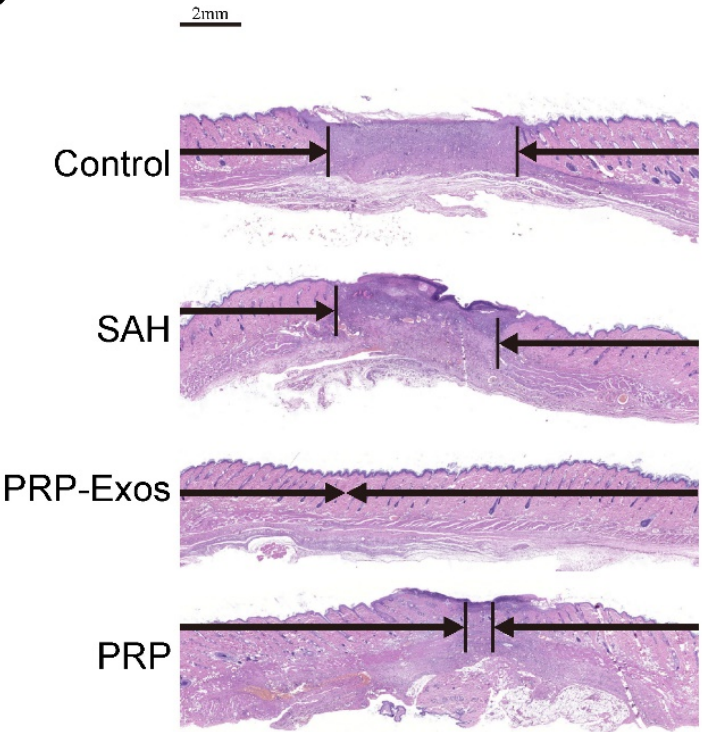

Figure 5. (A) Micro-CT evaluation of blood vessel formation in full-thickness skin defects left untreated (control) or treated with SAH, PRP or PRP-Exos at 14 days after surgery. Three-dimensional reconstructed images showing the new blood vessels. (B) Morphometric analysis of the new blood vessel area and the number of blood vessels. $* P<0.05$ compared with control. \#P $<0.05$ comparing between groups. (C) Transmitted light images of HE-stained sections of the untreated defects (control) and the defects treated with $\mathrm{SAH}, \mathrm{PRP}$ or PRP-Exos at 14 days after operation (scale bar $=2 \mathrm{~mm}$ ). The total width of the image represents the initial defect size $(1.8 \mathrm{~cm})$ while the black arrows indicate the neo-epithelium. (D) Total neo-epithelium length in the skin defects left untreated (control), treated with SAH, PRP or PRP-Exos at 14 days after operation. *P < 0.05 compared with control. \#P< 0.05 between PRP-Exos and PRP. 
trichrome-stained sections revealed differences in the repair efficiency of the wounds among all four treatment groups at different time points (Fig. 6). When the wound was compared to that of untreated wounds in depth, tremendous collagen deposition and large wavy collagen fibers were observed in the defects treated with PRP or PRP-Exos. Photographs of the wounds treated with PRP-Exos revealed larger amounts of collagen fibers that were arranged in a well-organized matrix similar to that of normal skin, indicating the enhancing effect of the PRP-Exos on collagen remodeling and epithelium regeneration. Generally speaking, there were more constructions that resembled hair follicles and sebaceous glands in the defects treated with PRP-Exos than in those treated with PRP, SAH or in the untreated group.

IHC staining for CD31 and IF staining for CD31 and a-SMA revealed that the number of newly-formed and mature blood vessels in the dermal defect increased from post-operative day 7 to day 14 (Fig. 7A). Quantification of the density of new blood vessels, defined as the number of positive CD31-stained cells per $\mathrm{mm}^{2}$, verified the increase in vessel number (Fig. 7B). At day 14, the defects treated with PRP-Exos had a significantly higher blood vessel density compared to the other three groups. Quantification of the density of mature blood vessels also showed an increase from post-operative day 7 to day 14 in all four treatment groups (Fig. 7B), but the values were lower than those of new blood vessels.
The number of mature vessels in the defects treated with PRP-Exos was significantly higher than in the defects treated with PRP or SAH or the untreated wounds at 14 days after operation.

\section{Discussion}

The effect of PRP on wound healing has been mainly attributed to release of growth factors from platelets [13]. However, molecular evidence for the action of PRP remains unclear. Recently, it has been suggested that besides growth factors, extracellular vesicles (EVs), including exosomes and microvesicles, may play a major role in cell-to-cell communication [19]. PRP activity might also be due to the efficient intercellular communication of bioactive molecules, mediated by nanosized vesicles, identified as exosomes [17]. Based on these considerations, the aim of this study was to investigate whether exosomes can be considered as effectors of PRP functions and to identify their related molecular mechanisms.

Since PRP is a concentration of platelets with growth factors which play an important role in wound healing [13], the effect of PRP is also partly mediated by free growth factors released from platelets. Among these factors, PDGFBB, VEGF, bFGF, and TGF- $\beta$ have the closest association with wound repair [6, 14], as they are involved in regeneration and are potential regulators of HMEC-1[9] and fibroblasts [2].

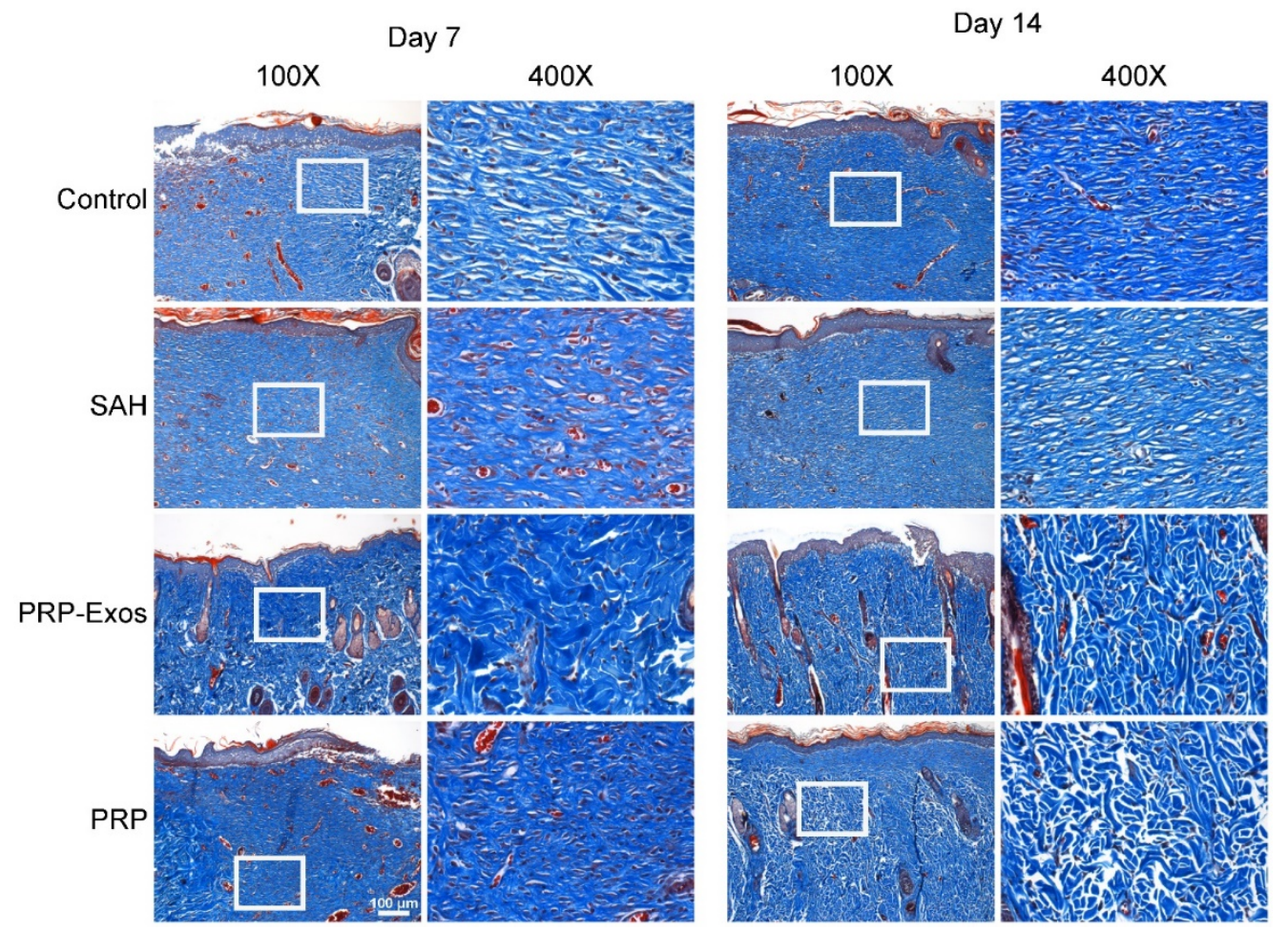

Figure 6. Transmitted light images of Masson's trichrome-stained sections of the untreated defects (control) and the defects treated with SAH, PRP or PRP-Exos at 7 and 14 days after operation, showing collagen deposition. Scale bar: $100 \mu \mathrm{m}$. 
A

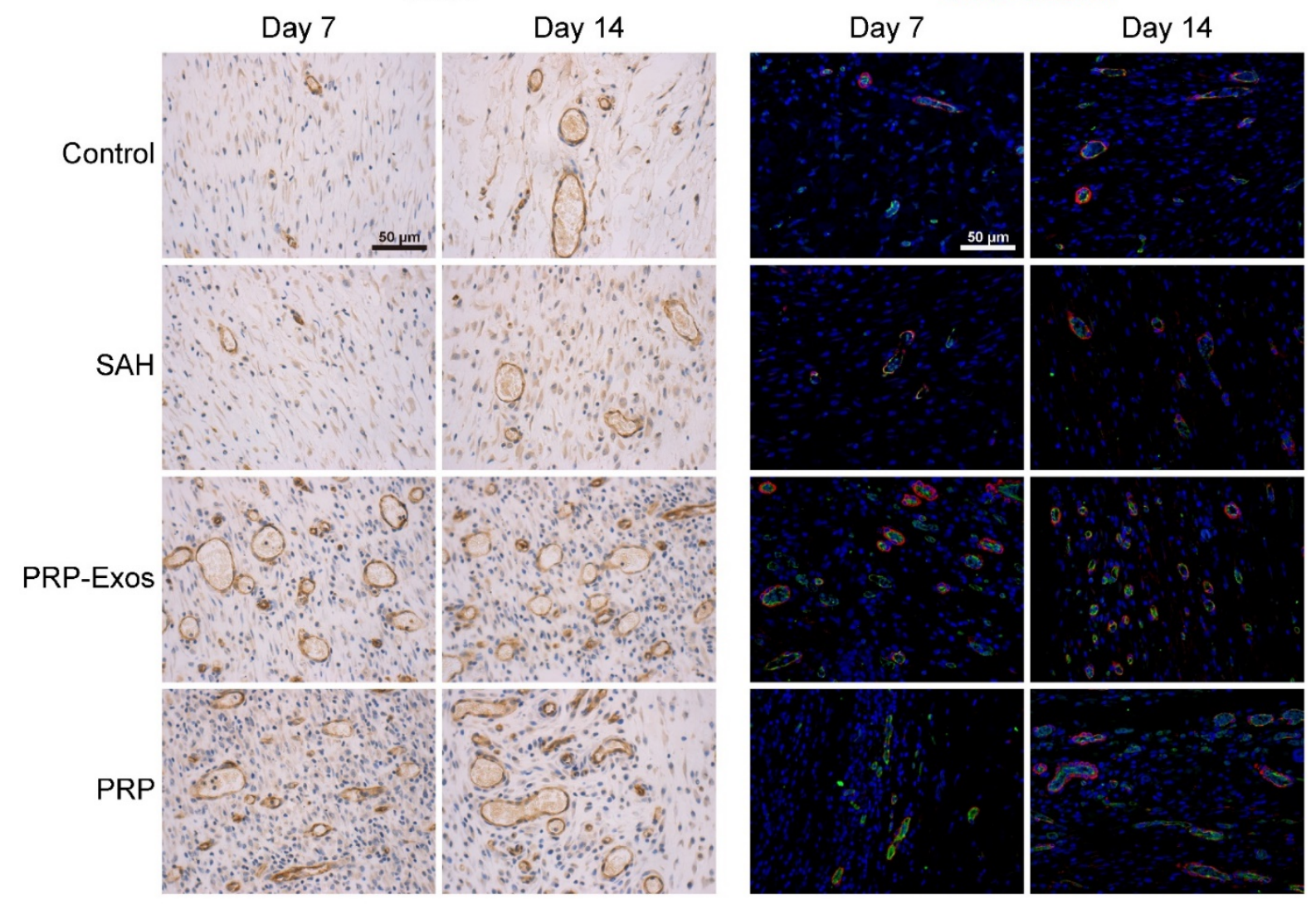

B
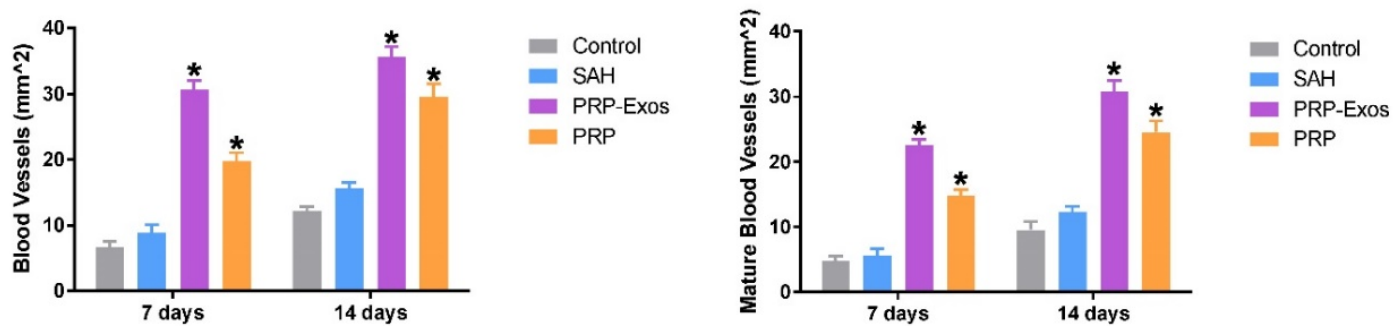

Figure 7. (A) IHC staining of CD31 (left) and IF staining of CD31 and a-SMA (right). Newly-formed blood vessels were identified by positive CD31 staining and their typical round or oval structure. ECs (CD31), smooth muscle cells $(\alpha-S M A)$ and cell nuclei are stained green, red and blue, respectively. Red and green co-staining indicates mature blood vessels. (B) Number of newly-formed blood vessels and mature blood vessels in the untreated defects (control) and the defects treated with SAH, PRP or PRP-Exos at 7 and 14 days after operation. Scale bar: $50 \mu \mathrm{m}$. $* P<0.05$ compared with control.

Recent studies have described the expression of growth factors in association with the exosome membrane [10]. Based on this evidence, we chose to test these growth factors, because they have been extensively reported to be actively involved in angiogenesis [14, 15]. Moreover, recombinant bFGF, PDGFBB and VEGF have all been shown to promote wound healing $[15,16]$. Interestingly, our results showed that theses growth factors can exist encapsulated within exosomes and demonstrated a significant enrichment of bFGF, PDGFBB, and TGF- $\beta$ expression in PRP-Exos compared to PRP-AS, suggesting a possible physiological role for PRP-Exos as a means of delivering these growth factors across the extracellular space $[17,24]$. In addition to this, characterization of the content of PRP-Exos at the protein level may provide detailed information on the molecular mechanisms involved. Based on published data, we treated HMEC-1 cells with different exosome concentrations and compared this to treatment with PRP-AS, and we were rather surprised to find that the differences between PRP-Exos and PRP-AS was significant in terms of proliferation rate, migration, and tube formation in vitro. Similar exosome effects have also been observed in diabetic rat models, indicating that a cocktail of factors contained in exosomes can boost cell proliferation and migration. All these data point to the fact that the angiogenesis-promoting effect of PRP is derived from its exosomes and indicate that exosomes possess the 
same potential to stimulate HMEC-1 growth as that provided by PRP [17]. At the same total protein content, the PRP-Exos showed greater promotional effects on cell proliferation and migration, which implies that the effective factors contained in exosomes are more enriched than in PRP [47].

In particular, megakaryocytes (MKs) are platelet progenitor cells, whose principal role is to maintain the normal blood platelet count and express most platelet proteins [12]. Platelets have little or no capacity to manufacture proteins. An unambiguous criterion for the identification of MKs is described as the high expression of platelet glycoproteins such as CD41 or CD61. The results of PRP-Exos protein expression analysis showed that the platelet marker CD41 was also expressed in the exosomes, confirming that the exosomes were derived from platelets.

Wound healing begins with fibroblast proliferation [2]. The repair process is activated when platelets come into contact with exposed collagen leading to platelet aggregation and the release of clotting factors, resulting in the deposition of a fibrin clot at the site of injury. The fibrin clot serves as a provisional matrix and sets the stage for the subsequent events of healing. The fibroblasts are the connective tissue cells responsible for collagen deposition required to repair the tissue injury. The collagen is the main constituent of extracellular tissue, which is responsible for support and strength. We isolated exosomes from human PRP and evaluated their potential effect on fibroblast proliferation and migration compared to PRP-AS. Fibroblasts cultured with PRP-Exos showed significant increases in proliferation and migration compared to PRP-AS. The enhanced content of TGF- $\beta$ in PRP-Exos may account for the re-epithelialization activity. From these results, we deduced that the PRP-Exos-induced promotion was duplicated when S127A was over-expressed in fibroblasts and blocked when YAP was knocked down. All these results demonstrated that YAP is the key regulatory point of PRP-Exos activity. Furthermore, the Y-27632 $2 \mathrm{HCl}$ inhibition of the promotional effect of PRP-Exos indicated that YAP activation was modulated by Rho. Following PRP-Exos treatment, YAP was de-phosphorylated, allowing it to translocate to the nucleus, activate the YAP downstream functional protein and participate in wound repair. With YAP activated, fibroblast migration was greatly increased, promoting a more rapid wound closure. At the same time, fibroblast proliferation was increased greatly through YAP de-phosphorylation and increased CTGF secretion. As fibroblast proliferation increased, collagen synthesis and deposition also increased [48]. When the skin attachment was formed, re-epithelialization was considered to be promoted [49]. Therefore, we confirmed that the wound-healing property of PRP-Exos is mediated by increasing collagen synthesis through YAP activation.

At the same time, the main angiogenesis-related signal pathway was tested at the protein level. The results showed that Erk and Akt were both phosphorylated to activate the angiogenesis signaling pathways. Through these two important pathways $[27,50,51]$, vascularization was greatly improved, with rapid promotion of the formation of granulation tissue, thus increasing the blood supply to the wound site.

Since sodium alginate has been used in wound dressing, tissue engineering, and drug delivery [52], different types of alginate-based wound dressing material have been commercialized and they have been widely reviewed [53]. So as to control the bioavailability of PRP-derived growth factors [54, 55] and achieve efficient delivery of the PRP-derived exosomes, a sodium alginate hydrogel carrier system was chosen even though PRP can be used directly in connective tissue graft procedures. The efficacy of topical platelet gel application has been evaluated in clinical studies in humans and a significant improvement in wound healing was observed.

Exosomes, one form of extracellular vesicles, have potential as mediators of intercellular communication even across species, and in fact cross-species communication has been reported several times. Extracellular vesicles derived from LX2, a human liver stellate cell line, have been used in a rat model [56], while therapeutic effects of human MSC-derived extracellular vesicles have been verified using a mouse [57] and a rat model [58]. Humans can absorb cow's milk exosomes and transport them to peripheral tissues across species boundaries [59]. The immune evasion mechanisms of exosomes remain elusive and will require further study. Our current hypothesis assumes that most protein was encapsulated within the lipid bilayers and exposed only during the exosome-cytomembrane interaction. The exposed protein quickly produced its effects, before being endocytosed and degraded by target cells.

In this study, we have demonstrated that a substantial part of the proliferation-, migration- and vessel formation-promoting effects of PRP is mediated by exosomes. In particular, this is the first time that the exact nature of the signals involved in these processes and how PRP-Exos are involved in their delivery, as well as the mechanism of action, has been studied in depth. 


\section{Summary and Conclusions}

In the present study, we demonstrate that exosomes can be successfully isolated and purified from human PRP. PRP-Exos increase proliferation and migration of HMEC- 1 cells and fibroblasts to a greater extent than PRP. In addition to this, preliminary analysis of exosome content showed that PRP-Exos encapsulated bFGF, PDGF-BB, VEGF, and TFG- $\beta$. Our findings indicate that exosomes released by PRP may contribute to PRP-induced angiogenesis through activation of Erk and Akt signaling pathways, and suggest that PRP-induced re-epithelialization may be triggered by activation of YAP.

\section{Abbreviations}

PRP: platelet-rich plasma

PRP-Exos: exosomes derived from PRP

YAP: yes-associated protein

HE stain: hematoxylin and eosin stain

ECM: extra-cellular matrix

BMSCs: bone mesenchymal stem cells

PS: penicillin-streptomycin solution

PBS: phosphate-buffered saline

DMEM: Dulbecco's modified Eagle's medium

FBS: fetal bovine serum

DLS: dynamic light scattering

TEM: transmission electron microscope

VEGF: vascular endothelial growth factor

bFGF: basic fibroblast growth factor

PDGF: platelet derived growth factor

TGF- $\beta$ : transforming growth factor- $\beta$

CTGF: connective tissue growth factor

Erk: extracellular signal regulated kinase

ROCK: Rho-associated coil kinase

ACD-A: acid citrate dextrose solution A

HMEC-1: a human microvascular endothelial cell line

IHC: immunohistochemistry

IF: immunofluorescence

SA: sodium alginate

CCK-8: cell counting kit-8

HRP: horseradish peroxidase

STZ: streptozotocin

CT: computed tomography

SAH: sodium alginate hydrogel

\section{Acknowledgements}

This study was supported by the National Natural Science Foundation of China (Grant No. 81301589, 81572239 and 81472066).

\section{Ethics committee approval and patient consent}

This study was in adherence with the principles of the Declaration of Helsinki. Written informed consent was obtained from each donor with the permission of the Institutional Review Board at Shanghai Sixth People's Hospital. The use of animals in these experiments followed the Interdisciplinary Principles and Guidelines for the Use of Animals in Research, Testing, and Education. All experimental and animal care procedures were approved by the Animal Research Ethics Committee of Shanghai Sixth People's Hospital and were performed in accordance with the guidelines of the National Institutes of Health Guidelines for the Care and Use of Laboratory Animals.

\section{Author contributions}

Shang-Chun Guo participated in design of the study, performed the statistical analysis and drafted the manuscript. Shi-Cong Tao performed the in vitro experiments, helped to draft the manuscript and performed the statistical analysis. Wen-Jing Yin helped with the animal experiments. Xin Qi participated in the animal experiments. Ting Yuan participated in design of the study. Chang-Qing Zhang conceived of the study and participated in its design. All authors read and approved the final manuscript.

\section{Competing interests}

The authors have declared that no competing interest exists.

\section{References}

1. Khamaisi M, Katagiri S, Keenan H, Park K, Maeda Y, Li Q, et al. PKCdelta inhibition normalizes the wound-healing capacity of diabetic human fibroblasts. J Clin Invest. 2016; 126: 837-53.

2. Driskell RR, Lichtenberger BM, Hoste E, Kretzschmar K, Simons BD, Charalambous $M$, et al. Distinct fibroblast lineages determine dermal architecture in skin development and repair. Nature. 2013; 504: 277-81.

3. Falanga V. Wound healing and its impairment in the diabetic foot. Lancet. 2005; 366: 1736-43

4. Demidova-Rice TN, Hamblin MR, Herman IM. Acute and impaired wound healing: pathophysiology and current methods for drug delivery, part 1: normal and chronic wounds: biology, causes, and approaches to care. Adv Skin Wound Care. 2012; 25: 304-14.

5. Nowell CS, Odermatt PD, Azzolin L, Hohnel S, Wagner EF, Fantner GE, et al. Chronic inflammation imposes aberrant cell fate in regenerating epithelia through mechanotransduction. Nat Cell Biol. 2016; 18: 168-80.

6. Carvajal-Gonzalez JM, Roman AC, Cerezo-Guisado MI, Rico-Leo EM, Martin-Partido G, Fernandez-Salguero PM. Loss of dioxin-receptor expression accelerates wound healing in vivo by a mechanism involving TGFbeta. J Cell Sci. 2009; 122: 1823-33.

7. Werdin F, Tenenhaus M, Rennekampff HO. Chronic wound care. Lancet. 2008; 372: $1860-2$.

8. Masiero FS, Thyssen PJ. Evaluation of conventional therapeutic methods versus maggot therapy in the evolution of healing of tegumental injuries in Wistar rats with and without diabetes mellitus. Parasitol Res. 2016; 115: 2403-7.

9. Icli B, Nabzdyk CS, Lujan-Hernandez J, Cahill M, Auster ME, Wara AK, et al. Regulation of impaired angiogenesis in diabetic dermal wound healing by microRNA-26a. J Mol Cell Cardiol. 2016; 91: 151-9.

10. Fontana F, Mori M, Riva F, Makila E, Liu D, Salonen J, et al. Platelet Lysate-Modified Porous Silicon Microparticles for Enhanced Cell Proliferation in Wound Healing Applications. ACS Appl Mater Interfaces. 2016; 8: 988-96. 
11. Zhang J, Yuan T, Wang JH. Moderate treadmill running exercise prior to tendon injury enhances wound healing in aging rats. Oncotarget. 2016; 7: 8498-512.

12. Geddis AE, Kaushansky K. Immunology. The root of platelet production. Science. 2007; 317: 1689-91.

13. Yuan T, Guo SC, Han P, Zhang CQ, Zeng BF. Applications of leukocyte- and platelet-rich plasma (L-PRP) in trauma surgery. Curr Pharm Biotechnol. 2012; 13: 1173-84.

14. Martino MM, Tortelli F, Mochizuki M, Traub S, Ben-David D, Kuhn GA, et al. Engineering the growth factor microenvironment with fibronectin domains to promote wound and bone tissue healing. Sci Transl Med. 2011; 3: 100ra89.

15. Losi P, Briganti E, Errico C, Lisella A, Sanguinetti E, Chiellini F, et al. Fibrin-based scaffold incorporating VEGF- and bFGF-loaded nanoparticles stimulates wound healing in diabetic mice. Acta Biomater. 2013; 9: 7814-21.

16. Beanes SR, Dang C, Soo C, Ting K. Skin repair and scar formation: the central role of TGF-beta. Expert Rev Mol Med. 2003; 5: 1-22.

17. Torreggiani E, Perut F, Roncuzzi L, Zini N, Baglio SR, Baldini N. Exosomes: novel effectors of human platelet lysate activity. Eur Cell Mater. 2014; 28: 137-51; discussion 51.

18. Montecalvo A, Larregina AT, Morelli AE. Methods of analysis of dendritic cell-derived exosome-shuttle microRNA and its horizontal propagation between dendritic cells. Methods Mol Biol. 2013; 1024: 19-40.

19. Kosaka N, Yoshioka Y, Hagiwara K, Tominaga N, Ochiya T. Functional analysis of exosomal microRNA in cell-cell communication research. Methods Mol Biol. 2013; 1024: 1-10.

20. Mittelbrunn M, Sanchez-Madrid F. Intercellular communication: diverse structures for exchange of genetic information. Nat Rev Mol Cell Biol. 2012; 13: 328-35.

21. Huber HJ, Holvoet P. Exosomes: emerging roles in communication between blood cells and vascular tissues during atherosclerosis. Curr Opin Lipidol. 2015; 26: 412-9.

22. Melo SA, Luecke LB, Kahlert C, Fernandez AF, Gammon ST, Kaye J, et al. Glypican-1 identifies cancer exosomes and detects early pancreatic cancer. Nature. 2015; 523: 177-82.

23. Alexander M, Hu R, Runtsch MC, Kagele DA, Mosbruger TL, Tolmachova T, et al. Exosome-delivered microRNAs modulate the inflammatory response to endotoxin. Nat Commun. 2015; 6: 7321.

24. Mistry DS, Chen Y, Sen GL. Progenitor function in self-renewing human epidermis is maintained by the exosome. Cell Stem Cell. 2012; 11: 127-35.

25. Burgess DJ. Signalling: vesicle vehicles of genetic information. Nat Rev Genet. 2014; 15: 514.

26. Robbins PD, Morelli AE. Regulation of immune responses by extracellular vesicles. Nat Rev Immunol. 2014; 14: 195-208.

27. Ruan GX, Kazlauskas A. Axl is essential for VEGF-A-dependent activation of PI3K/Akt. EMBO J. 2012; 31: 1692-703.

28. Zhu H, Cheng X, Niu X, Zhang Y, Guan J, Liu X, et al. Proton-sensing GPCR-YAP Signalling Promotes Cell Proliferation and Survival. Int J Biol Sci. 2015; 11: 1181-9.

29. Zhu H, Guo S, Zhang Y, Yin J, Yin W, Tao S, et al. Proton-sensing GPCR-YAP Signalling Promotes Cancer-associated Fibroblast Activation of Mesenchymal Stem Cells. Int J Biol Sci. 2016; 12: 389-96.

30. Tao SC, Gao YS, Zhu HY, Yin JH, Chen YX, Zhang YL, et al. Decreased extracellular $\mathrm{pH}$ inhibits osteogenesis through proton-sensing GPR4-mediated suppression of yes-associated protein. Sci Rep. 2016; 6: 26835.

31. Schlegelmilch K, Mohseni M, Kirak O, Pruszak J, Rodriguez JR, Zhou D, et al. Yap1 acts downstream of alpha-catenin to control epidermal proliferation. Cell. 2011; 144: 782-95.

32. Wakefield LM, Stuelten C. Keeping order in the neighborhood: new roles for TGFbeta in maintaining epithelial homeostasis. Cancer Cell. 2007; 12: 293-5.

33. Lawson $\mathrm{CD}$, Burridge $\mathrm{K}$. The on-off relationship of Rho and Rac during integrin-mediated adhesion and cell migration. Small GTPases. 2014; 5: e27958.

34. Yi L, Huang X, Guo F, Zhou Z, Dou Y, Huan J. Yes-associated protein (YAP) signaling regulates lipopolysaccharide-induced tissue factor expression in human endothelial cells. Surgery. 2016; 159: 1436-48.

35. Huang J, Wu S, Barrera J, Matthews K, Pan D. The Hippo signaling pathway coordinately regulates cell proliferation and apoptosis by inactivating Yorkie, the Drosophila Homolog of YAP. Cell. 2005; 122: 421-34.

36. Yuan T, Zhang C, Zeng B. Treatment of chronic femoral osteomyelitis with platelet-rich plasma (PRP): a case report. Transfus Apher Sci. 2008; 38: 167-73.

37. Yin $\mathrm{W}$, Qi X, Zhang Y, Sheng J, Xu Z, Tao S, et al. Advantages of pure platelet-rich plasma compared with leukocyte- and platelet-rich plasma in promoting repair of bone defects. J Transl Med. 2016; 14: 73.

38. Thery C, Amigorena S, Raposo G, Clayton A. Isolation and characterization of exosomes from cell culture supernatants and biological fluids. Curr Protoc Cell Biol. 2006; Chapter 3: Unit 322.

39. Yang $\mathrm{X}$, Meng $\mathrm{S}$, Jiang $\mathrm{H}$, Zhu $\mathrm{C}, \mathrm{Wu} \mathrm{W}$. Exosomes derived from immature bone marrow dendritic cells induce tolerogenicity of intestinal transplantation in rats. J Surg Res. 2011; 171: 826-32.

40. Rnjak J, Li Z, Maitz PK, Wise SG, Weiss AS. Primary human dermal fibroblast interactions with open weave three-dimensional scaffolds prepared from synthetic human elastin. Biomaterials. 2009; 30: 6469-77.

41. Blandino A, Macias M, Cantero D. Formation of calcium alginate gel capsules: influence of sodium alginate and $\mathrm{CaCl} 2$ concentration on gelation kinetics. J Biosci Bioeng. 1999; 88: 686-9.
42. Iwakura A, Tabata $Y$, Tamura N, Doi K, Nishimura $K$, Nakamura $T$, et al. Gelatin sheet incorporating basic fibroblast growth factor enhances healing of devascularized sternum in diabetic rats. Circulation. 2001; 104: I325-9.

43. Zhao S, Li L, Wang H, Zhang Y, Cheng X, Zhou N, et al. Wound dressings composed of copper-doped borate bioactive glass microfibers stimulate angiogenesis and heal full-thickness skin defects in a rodent model. Biomaterials. 2015; 53: 379-91.

44. Di Benedetto A, Mottolese M, Sperati F, Ercolani C, Di Lauro L, Pizzuti L, et al. The Hippo transducers TAZ/YAP and their target CTGF in male breast cancer. Oncotarget. 2016.

45. Holmes A, Abraham DJ, Sa S, Shiwen X, Black CM, Leask A. CTGF and SMADs, maintenance of scleroderma phenotype is independent of SMAD signaling. J Biol Chem. 2001; 276: 10594-601.

46. Sagi I, Chia G, Golan-Lev T, Peretz M, Weissbein U, Sui L, et al. Derivation and differentiation of haploid human embryonic stem cells. Nature. 2016; 532: 107-11.

47. Lopatina T, Bruno S, Tetta C, Kalinina N, Porta M, Camussi G. Platelet-derived growth factor regulates the secretion of extracellular vesicles by adipose mesenchymal stem cells and enhances their angiogenic potential. Cell Commun Signal. 2014; 12: 26.

48. Lo Re S, Lecocq M, Uwambayinema F, Yakoub Y, Delos M, Demoulin JB, et al. Platelet-derived growth factor-producing CD4+ Foxp3+ regulatory $\mathrm{T}$ lymphocytes promote lung fibrosis. Am J Respir Crit Care Med. 2011; 184: $1270-81$

49. Cerqueira MT, da Silva LP, Santos TC, Pirraco RP, Correlo VM, Reis RL, et al. Gellan gum-hyaluronic acid spongy-like hydrogels and cells from adipose tissue synergize promoting neoskin vascularization. ACS Appl Mater Interfaces. 2014; 6: 19668-79.

50. Vanhaesebroeck B, Stephens L, Hawkins P. PI3K signalling: the path to discovery and understanding. Nat Rev Mol Cell Biol. 2012; 13: 195-203.

51. Gentilini D, Busacca M, Di Francesco S, Vignali M, Vigano P, Di Blasio AM. PI3K/Akt and ERK1/2 signalling pathways are involved in endometrial cell migration induced by 17 beta-estradiol and growth factors. Mol Hum Reprod. 2007; $13: 317-22$

52. Zhang J, Wang Q, Wang A. In situ generation of sodium alginate/hydroxyapatite nanocomposite beads as drug-controlled release matrices. Acta Biomater. 2010; 6: 445-54.

53. Ruvinov E, Cohen S. Alginate biomaterial for the treatment of myocardial infarction: Progress, translational strategies, and clinical outlook: From ocean algae to patient bedside. Adv Drug Deliv Rev. 2016; 96: 54-76.

54. Lin SS, Landesberg R, Chin HS, Lin J, Eisig SB, Lu HH. Controlled release of PRP-derived growth factors promotes osteogenic differentiation of human mesenchymal stem cells. Conf Proc IEEE Eng Med Biol Soc. 2006; 1: 4358-61.

55. Lu HH, Vo JM, Chin HS, Lin J, Cozin M, Tsay R, et al. Controlled delivery of platelet-rich plasma-derived growth factors for bone formation. J Biomed Mater Res A. 2008; 86: 1128-36.

56. Li L, Piontek K, Ishida M, Fausther M, Dranoff JA, Fu R, et al. Extracellular vesicles carry miR-195 to intrahepatic cholangiocarcinoma and improve survival in a rat model. Hepatology. 2016

57. Monsel A, Zhu YG, Gennai S, Hao Q, Hu S, Rouby JJ, et al. Therapeutic Effects of Human Mesenchymal Stem Cell-derived Microvesicles in Severe Pneumonia in Mice. Am J Respir Crit Care Med. 2015; 192: 324-36.

58. Zhang B, Wang M, Gong A, Zhang X, Wu X, Zhu Y, et al. HucMSC-Exosome Mediated-Wnt 4 Signaling Is Required for Cutaneous Wound Healing. Stem Cells. 2015; 33: 2158-68.

59. Kusuma RJ, Manca S, Friemel T, Sukreet S, Nguyen C, Zempleni J. Human vascular endothelial cells transport foreign exosomes from cow's milk by endocytosis. Am J Physiol Cell Physiol. 2016; 310: C800-7. 